'Departamento de Matemática y

Estadística, Facultad de Ciencias Bioquímicas y Farmacéuticas, Universidad Nacional de Rosario. Santa $\mathrm{Fe}$, Argentina.

2Área Farmacología, Facultad de Ciencias Bioquímicas y Farmacéuticas, Universidad Nacional de Rosario. Santa $\mathrm{Fe}$, Argentina.

${ }^{3}$ Cátedra de Farmacología, Facultad de Medicina, Universidad Nacional de Buenos Aires. Buenos Aires, Argentina.

${ }^{4}$ Área Tecnología en Salud Pública,

Facultad de Ciencias Bioquímicas y

Farmacéuticas, Universidad Nacional de Rosario. Santa Fe. Argentina. aBioquímica, Farmacéutica.

${ }^{\text {b} F a r m a c e ́ u t i c a . ~}$ 'Doctora (PhD).

${ }^{\mathrm{d}}$ Doctora en Ciencias Biomédicas (PhD).

Fuentes de apoyo financiero: $\mathrm{El}$ proyecto que dio origen a este trabajo fue subsidiado por concurso por las siguientes entidades:-Gobierno de la Provincia de Santa Fe, en el contexto del "Programa de fortalecimiento

de las capacidades del sistema de investigación y desarrollo en la provincia de Santa Fe". - Secretaría de Extensión de la Universidad Nacional de Rosario en el marco de la 3 a Convocatoria "La Universidad y su compromiso con la Sociedad". Las organizaciones mencionadas no tuvieron influencia en ninguna de las etapas del trabajo realizado.

Recibido el 19 de mayo de 2012, aceptado el 25 de septiembre de 2012

Correspondencia a: Marta Mónica Marzi. Dirección postal: Presidente Julio Roca, No 653, Piso 7, Dpto. A. Rosario, Santa Fe, Argentina. Teléfonos: 03414253300.

E-mail: marzimarta58@gmail.com

\section{Análisis de la prescripción de medicamentos en una comunidad geriátrica Argentina}

\author{
MARTA MARZI M. ${ }^{1, \mathrm{a}}$, VALERIANA DIRUSCIO A. ${ }^{2, \mathrm{~b}}$, \\ MARIANO NÚÑEZ H. ${ }^{3}$, MIRYAM PIRES S., ${ }^{4, c}$, NORA QUAGLIA B. ${ }^{2, d}$
}

Background: Although polypharmacy may be justified in elderly patients with multiple diseases, it may be dangerous, especially when it includes potentially inappropriate medications (PIM). Aim: To identify inappropriate medication and factors associated with the most relevant prescriptions among older people. Material and Methods: Cross-sectional observational analysis of drugs prescribed during the first trimester of 2010 to 179 older adults aged $77 \pm 8$ years ( 98 women), living in a geriatric reference hospital in Argentina. The use of potentially inappropriate medications (PIM) in elderly patients was analyzed using Beers Criteria updated to 2012. Results: The mean number of drugs prescribed per individual was $6.1 \pm 2.7$. The most commonly used drugs were anti-ulcer agents (58.1\%), agents acting on the renin-angiotensin system (54.2\%), antithrombotic medications (50.8\%) and benzodiazepines (50.8\%). The use of antacids and anti-ulcer agents, psychotropic drugs and PIM was significantly higher among patients using six or more drugs daily, compared to the less medicated group (odds ratio (OR) $=6.8,95 \%$ confidence intervals $(C I) 3.5-13.2 ; \mathrm{OR}=15.0,95 \%$ CI 5.9-38.4; $O R=5.0 ; 95 \% I C$ 2.6-9.8, respectively). Thirty one percent of participants using non-steroidal anti-inflammatory drugs, were not receiving medications for gastric protection. One to four drugs included in the Beers list were prescribed to $66 \%$ of participants. Conclusions: Despite the high prevalence of use of antacids and anti-ulcer agents, these drugs were not prescribed to a significant proportion of patients using non-steroidal anti-inflammatory drugs. Strategies to optimize pharmacotherapy in the elderly population are urgently required.

(Rev Med Chile 2013; 141: 194-201).

Key words: Aged; Errors; Inappropriate prescribing; Polypharmacy.

41 idac l progresivo envejecimiento de la población y los cambios epidemiológicos de las enfermedades están produciendo importantes retos sanitarios. El predominio de enfermedades crónicas y de pluripatología requiere el uso de muchas drogas para un apropiado tratamiento ocasionando un incremento de la demanda de los recursos sanitarios y condicionando la práctica asistencial.

La referencia a la utilización de múltiples medicamentos es frecuentemente conocida como polifarmacia, pero en un sentido más estricto se ha postulado que la polifarmacia es la administración de más medicamentos de los que son clínicamente necesarios ${ }^{1}$. 
El incremento de la edad del individuo está asociado con cambios en el organismo que pueden afectar la distribución, el metabolismo y la excreción de las drogas, razón por la que los adultos mayores constituyen una población susceptible de sufrir eventos adversos a drogas ${ }^{2-4}$.

Los medicamentos cuyo riesgo de eventos adversos en los adultos mayores excede expectativas de beneficios clínicos existiendo alternativas más efectivas y seguras son llamados medicamentos potencialmente inapropiados $(\mathrm{MPI})^{5}$. Se ha reportado que la polifarmacia incrementa el riesgo de consumir MPI y de que existan interacciones entre drogas ${ }^{4}$. Beers y col. elaboraron Criterios de MPI que deberían evitarse en ancianos, la última actualización de estos criterios fue publicada en el año $2012^{6}$.

El objetivo de este trabajo fue determinar el perfil de prescripciones de medicamentos en la población de adultos mayores de una residencia pública de referencia en la atención geriátrica, identificando la medicación inapropiada y los factores asociados a las prescripciones más relevantes.

\section{Material y Método}

Se realizó un estudio retrospectivo observacional de corte transversal sobre utilización de medicamentos en un centro público de atención geriátrica dependiente del Ministerio de Salud de la Provincia de Santa Fe. Se trata del Hospital Geriátrico Provincial de la ciudad de Rosario, centro de referencia en la provincia, donde el paciente reside y se le brinda atención integral especializada. Los fármacos prescriptos en dicha institución están incluidos en el Formulario Terapéutico Provincial, que es de uso obligatorio en las instituciones oficiales de la provincia. Dicho formulario es un listado de medicamentos esenciales basado en recomendaciones de la Organización Mundial de la Salud (OMS) y en listados nacionales e internacionales, no específico para adultos mayores.

Se recabó información de todos los residentes durante el primer trimestre del año 2010, a partir de las prescripciones médicas obtenidas en la oficina de farmacia del hospital. Se registró edad, sexo y drogas dispensadas con sus dosis respectivas. Durante el período estudiado residían en el hospital geriátrico 183 personas. De estas, se excluyeron 4 por tener menos de 60 años, quedando un total de 179 adultos mayores incluidos en el estudio.
Las drogas fueron clasificadas según el sistema Químico Terapéutico Anatómico (ATC) desarrollado por la $\mathrm{OMS}^{7}$, excepto en los siguientes casos y sólo con el fin de obtener mayor homogeneidad respecto al uso terapéutico de las mismas:

Clonazepam: si bien en el sistema ATC pertenece al grupo de los anticonvulsivantes (N03), en la población estudiada ha sido prescripto en dosis bajas, generalmente utilizadas para trastornos de ansiedad, razón por la que se lo incluyó en el grupo de las benzodiazepinas (N05B).

Zolpidén: fue incluido en el grupo de las benzodiazepinas.

El ácido acetilsalicílico como inhibidor de la agregación plaquetaria (B01AC06) fue incluido en el grupo de los agentes antitrombóticos (B01) si la dosis prescripta era menor o igual a $300 \mathrm{mg} /$ día.

Para el análisis del uso de MPI en ancianos se utilizó la actualización 2012 de los Criterios de Beers independientes del diagnóstico ${ }^{6}$. Se identificaron las drogas prescriptas que respondían a estos criterios y se calculó la prevalencia de utilización.

Las características basales fueron descriptas mediante números, porcentajes y medias con su desvío estándar (DE). Se realizó análisis bivariado y multivariado para analizar la asociación entre medicación inapropiada y medicamentos prevalentes con variables tales como género, edad y polifarmacia. Se identificaron dos grupos etarios, adultos con edad entre 60 y 79 años y adultos con 80 años o más, y dos grupos según la cantidad de medicamentos consumidos tomando como punto de corte la media de fármacos de la población en estudio. Las razones de probabilidades u odds ratio (OR) se obtuvieron con sus correspondientes intervalos de confianza de 95\% (95\%IC). El análisis estadístico se realizó con el programa Epi Info 3.4.3; se consideró significativo un valor de $\mathrm{p}<0,05$.

El proyecto del estudio fue aprobado por el Comité de Bioética de la Facultad de Ciencias Bioquímicas y Farmacéuticas de la Universidad Nacional de Rosario y autorizado por la Dirección Médica del Hospital Geriátrico Provincial.

\section{Resultados}

El estudio se realizó sobre 179 adultos mayores, 98 mujeres y 81 varones, con una media de edad de 76,7 años $(\mathrm{DE}=8,3 ;$ rango $=60-100$ años $)$. 


\section{Medicamentos prescriptos}

El número medio de medicamentos prescritos por persona para uso regular fue $6,1(\mathrm{DE}=2,7$; rango $=1-14)$, no encontrándose diferencias significativas según género. La edad se asoció con el consumo de fármacos observándose que, en el grupo de pacientes que utilizaba diariamente un número de medicamentos inferior a la media, los ancianos menores de 80 años tenían prescripto un número de fármacos que en promedio superaba al de los ancianos mayores $(3,9, \mathrm{DE}=1,3$ vs 3,2 , $\mathrm{DE}=1,4 ; \mathrm{p}=0,0240)$. En el grupo de pacientes que consumía regularmente 6 ó más fármacos la diferencia observada entre los grupos etarios no fue significativa.

Los grupos de fármacos más prescriptos fueron: agentes para el tratamiento de alteraciones causadas por secreción gástrica de ácido (A02), agentes que actúan sobre el sistema renina-angiotensina (C09), antitrombóticos y benzodiazepinas (Tabla 1). Ranitidina, enalapril, ácido acetilsalicílico en dosis menor o igual a $300 \mathrm{mg} /$ día (AASp) y clonazepam fueron los fármacos más utilizados de cada grupo, respectivamente (Figura 1).

En el primero de los grupos mencionados, A02, las prescripciones incluían ranitidina, omeprazol, sucralfato e hidróxidos de aluminio y de magnesio. De los 104 pacientes con indicación de A02,
$76(73,1 \%)$ utilizaron regularmente ranitidina, $22(21,2 \%)$ omeprazol y $6(5,8 \%)$ ranitidina y omeprazol.

El enalapril fue utilizado por 92,8\% (90/97) de los pacientes con indicación de un agente del grupo C09, el ASSp por 79,1\% (72/91) de los ancianos con antitrombóticos y el clonazepam por $67,3 \%$ (66/98) de los que utilizaban benzodiazepinas.

\section{Asociación entre los grupos de medicamentos más prescriptos y las variables estudiadas}

Se estudió la potencial asociación entre el consumo de fármacos del grupo A02 y la prescripción de analgésicos antiinflamatorias no esteroideos (AINEs) que incluía ibuprofeno, diclofenaco y naproxeno. Se halló que 68,9\% (31/45) de los pacientes que usaban AINEs tenía también indicado algún agente antiácido-antiulceroso. Esta relación porcentual no resultó diferente significativamente de la proporción de pacientes que no usando AINEs, utilizaba A02 (54,5\%, 73/134). Resultó, por el contrario, claramente significativa la asociación entre la utilización de paracetamol y/o analgésicos opioides y la indicación de fármacos del grupo A02 ( $\mathrm{p}=0,0023)$, así como también la relación entre la "chance" de utilizar A02 en el grupo de ancianos expuestos al consumo de un número de fármacos superior al promedio respecto al del

Tabla 1. Frecuencia de utilización de los 12 grupos de drogas más prescriptos $(n=179)$

\begin{tabular}{|lccc|}
\hline Grupos de drogas & Categoría ATC* & Pacientes & n \\
\hline Agentes para el tratamiento de alteraciones causadas por ácidos & A02 & 104 & 58,1 \\
\hline Agentes que actúan sobre el sistema renina-angiotensina & C09 & 97 & 54,2 \\
\hline Agentes antitrombóticos & B01 & 91 & 50,8 \\
\hline Benzodiazepinas** & - & 91 & 50,8 \\
\hline Antipsicóticos & N05A & 60 & 33,5 \\
\hline Antiepilépticos & N03A & 53 & 29,6 \\
\hline Antiinflamatorios y antirreumáticos no esteroideos & M01A & 45 & 25,1 \\
\hline Diuréticos & C03 & 43 & 24,0 \\
\hline Preparados antianémicos & B03 & 42 & 23,5 \\
\hline Bloqueantes de canales de calcio & C08 & 36 & 20,1 \\
\hline Vitaminas & A11 & 33 & 18,4 \\
\hline Drogas para el tratamiento de enfermedades óseas & M05 & 32 & 17,9 \\
\hline
\end{tabular}

*Sistema de Clasificación Químico Terapéutico Anatómico desarrollado por la Organización Mundial de la Salud. **Incluye Clonazepam y Zolpidem. 


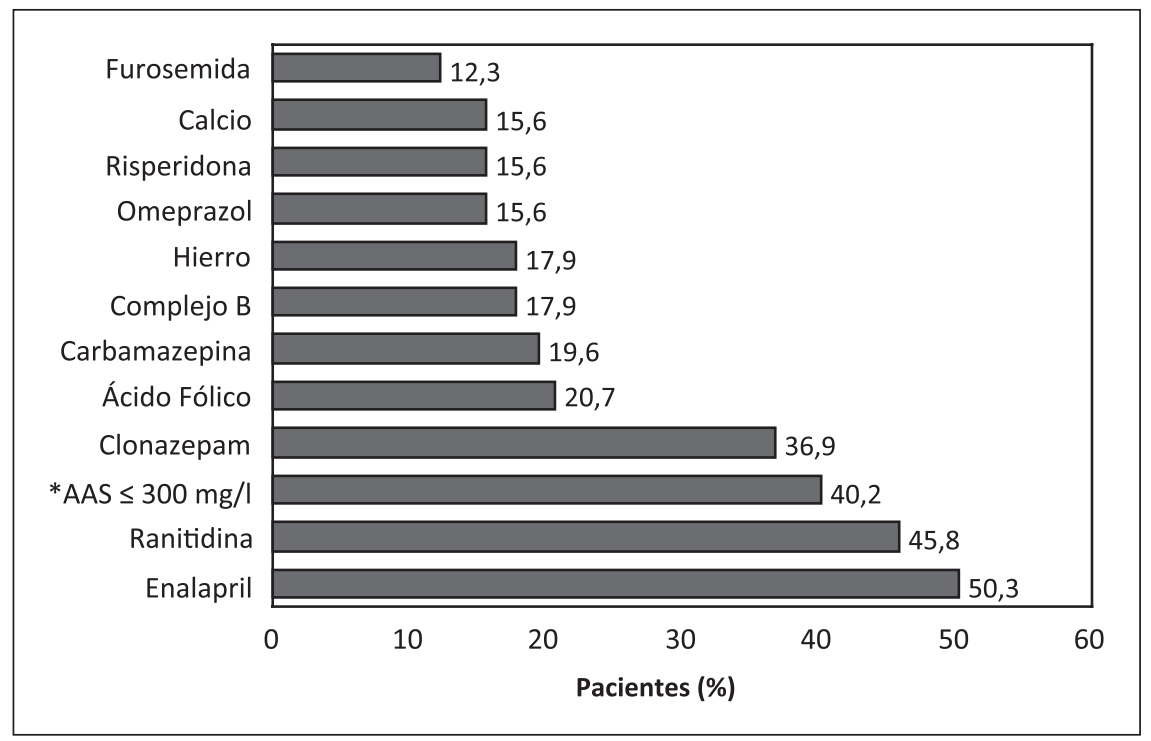

Figura 1. Frecuencia de utilización de los 12 fármacos más prescriptos $(n=179)$. *AAS: ácido acetilsalicílico. grupo con menos de 6 fármacos diarios $(\mathrm{OR}=6,8$; $95 \%$ IC $=3,5-13,2)$. Se comparó el grupo de pacientes usuarios de AINEs con el grupo usuario de paracetamol-analgésicos opioides en relación al número de fármacos consumidos obteniéndose un promedio por paciente de 6,5 $(\mathrm{DE}=2,4)$ para el primero y $8,8(\mathrm{DE}=2,8)$ para el segundo, $\mathrm{p}=0,0037$.

Los agentes del grupo C09 fueron utilizados por $67,3 \%$ (68/101) de los pacientes más medicados y por 37,2\% (29/78) del grupo con menos de 6 fármacos diarios $(p=0,0001)$, no encontrándose diferencias significativas según género ni grupo etario. El consumo de fármacos antitrombóticos no se asoció con ninguna de las variables analizadas.

El 75,4\% (135/179) de la población estudiada consumía en forma regular al menos un psicofármaco (rango $=1-5)($ Tabla 2$)$. Las benzodiazepinas, los antipsicóticos, los antiepilépticos y los antidepresivos constituyeron el 85,1\% de las prescripciones de drogas con acción sobre el sistema nervioso central (Tabla 3).

En el grupo de pacientes que consumía regularmente 6 o más fármacos diarios el 94,1\% (95/101) tenía prescripto al menos un psicotrópico, resultando esta prevalencia 1,8 veces superior a la del grupo con menos de 6 fármacos $(\mathrm{p}<0,0001)$. Para las benzodiazepinas, utilizada por $66,3 \%(67 / 101)$ de los ancianos polimedicados, la razón de preva- lencias entre ambos grupos fue 2,2 ( $\mathrm{p}<0,0001)$.

Entre los pacientes con indicación de un número de fármacos inferior a la media se encontró una diferencia estadísticamente significativa en la utilización de psicotrópicos en general y de benzodiazepinas en particular entre los ancianos más jóvenes en comparación con aquellos que contaban con 80 años o más. El OR de utilizar al menos un psicotrópico fue 2,6 veces mayor en los ancianos menores de 80 años que en los de más edad $(\mathrm{OR}=2,6,95 \% \mathrm{IC}=1,0-6,4)$. Para las benzodiazepinas dicha relación fue de $3,8(\mathrm{OR}=3,8$, $95 \%$ IC $=1,3-10,9)$.

Medicamentos potencialmente inapropiados para uso en los adultos mayores según la actualización 2012 de los Criterios de Beers.

Tabla 2. Frecuencia de utilización de psicofármacos $(n=179)$

\begin{tabular}{|ccc|}
\hline$N^{\circ}$ de psicofármacos & \multicolumn{2}{c|}{ Pacientes } \\
\hline 1 & 40 & $\%$ \\
\hline 2 & 46 & 22,3 \\
3 & 32 & 17,9 \\
4 & 13 & 7,3 \\
5 & 4 & 2,2 \\
Total & 135 & 75,4 \\
\hline
\end{tabular}


Tabla 3. Frecuencia de prescripción de drogas psicotrópicas

\begin{tabular}{|lcrr|}
\hline Grupos de drogas & $\begin{array}{c}\text { Categoría } \\
\text { ATC* }\end{array}$ & $\begin{array}{c}\text { Prescripciones } \\
\text { n }\end{array}$ & \multicolumn{1}{c}{$\%$} \\
Benzodiazepinas (BZD)** & - & 98 & 32,3 \\
\hline Analgésicos opioides & N02A & 10 & 3,3 \\
\hline Antiepilépticos & N03A & 60 & 19,8 \\
\hline Antiparkinsonianos anticolinérgicos & N04A & 5 & 1,7 \\
\hline Antiparkinsonianos dopaminérgicos & N04B & 14 & 4,6 \\
\hline Antipsicóticos & N05A & 77 & 25,4 \\
\hline Ansiolíticos (excluidas las BZD) & N05B & 3 & 1,0 \\
\hline Antidepresivos & N06A & 23 & 7,6 \\
\hline Drogas contra la demencia & N06D & 10 & 4,3 \\
\hline Total de prescripciones de psicotrópicos & & 300 & 100 \\
\hline
\end{tabular}

*Sistema de Clasificación Químico Terapéutico Anatómico desarrollado por la Organización Mundial de la Salud. **En el grupo de las BZD (N05B) se incluyó Zolpiden, droga relacionada con las benzodiazepinas (N05C) de acción sedante y Clonazepam, que si bien en el sistema ATC pertenece al grupo de los anticonvulsivantes (N03), en la población estudiada ha sido prescripto para trastornos de ansiedad.
De las 1.086 prescripciones de fármacos, 179 (16,5\%) fueron identificadas como potencialmente inapropiadas para uso en adultos mayores e incluían 18 drogas diferentes que fueron utilizadas por $65,9 \%(\mathrm{n}=118)$ de los ancianos en un rango de 1 a 4 (Tabla 4, Tabla 5). El OR de consumir estas drogas fue 5 veces mayor en los pacientes polimedicados que en los ancianos con menos de 6 fármacos diarios $(\mathrm{OR}=5,0 ; 95 \% \mathrm{IC}=2,6-9,8)$, con una media de MPI en cada grupo de $1,3(\mathrm{DE}=0,9)$ y $0,6(\mathrm{DE}=0,7)$ respectivamente $(\mathrm{p}<0,0001)$. No se observó consumo diferencial según género ni grupo etario.

Dentro de los psicofármacos utilizados, 7 se hallaron incluidos en la lista de Beers, tioridazina, haloperidol, clonazepam, lorazepam, diazepam, alplazolam y zolpidem. Del total de

Tabla 4. Medicamentos potencialmente inapropiados. Frecuencia de prescripción

\begin{tabular}{|c|c|c|c|c|}
\hline \multicolumn{2}{|c|}{ Drogas prescriptas incluidas en los criterios de Beers } & \multirow{3}{*}{$\begin{array}{c}\text { Códico ATC } \\
\text { D04AA32 }\end{array}$} & \multicolumn{2}{|c|}{ Prescripciones } \\
\hline & & & $\mathbf{n}$ & $\%$ \\
\hline \multirow[t]{5}{*}{ Drogas con efectos anticolinérgicos } & Difenhidramina & & 1 & 0,6 \\
\hline & Hidroxicina & N05BB01 & 3 & 1,7 \\
\hline & Prometazina & R06AD02 & 1 & 0,6 \\
\hline & Tioridazina & N05AC02 & 10 & 5,6 \\
\hline & Oxibutinina & G04BD04 & 6 & 3,4 \\
\hline \multirow[t]{5}{*}{ Benzodiazepinas } & Diazepam & N05BA01 & 9 & 5,0 \\
\hline & Clonazepam & N03AE01 & 66 & 36,9 \\
\hline & Lorazepam & NO5BAO6 & 15 & 8,4 \\
\hline & Alprazolam & N05BA12 & 2 & 1,1 \\
\hline & Zolpidem & N05CF02 & 5 & 2,8 \\
\hline \multirow[t]{3}{*}{ AINEs } & Naproxeno & M01AE02 & 7 & 3,9 \\
\hline & Ibuprofeno & M01AE01 & 19 & 10,6 \\
\hline & Diclofenaco & M01AB05 & 20 & 11,2 \\
\hline \multirow[t]{5}{*}{ Otras } & Haloperidol & N05AD01 & 7 & 3,9 \\
\hline & Nitrofurantoína & J01XE01 & 1 & 0,6 \\
\hline & Amiodarona & C01BD01 & 3 & 1,7 \\
\hline & Espironolactona $>25 \mathrm{mg} /$ día & C03DA01 & 3 & 1,7 \\
\hline & Digoxina $>0,125$ mg/día & C01AA05 & 1 & 0,6 \\
\hline Total & & & 179 & 100 \\
\hline
\end{tabular}




\section{Tabla 5. Frecuencia de utilización de medicamentos potencialmente inapropiados ( $n=179)$}

\begin{tabular}{|crc|}
\hline n de drogas & \multicolumn{1}{c|}{ Pacientes } \\
& n & \% \\
1 & 68 & 38,0 \\
2 & 40 & 22,3 \\
3 & 9 & 5,0 \\
4 & 1 & 0,6 \\
\hline Total & 118 & 65,9 \\
\hline
\end{tabular}

pacientes que utilizaron al menos un fármaco psicotrópico, 72,6\% (98/135) tenía prescripto alguno de los mencionados.

\section{Discusión}

Aproximadamente la mitad de las personas que residían en el Hospital Geriátrico consumía regularmente seis o más fármacos por día, este hallazgo es consistente con el reportado en estudios epidemiológicos de otros países ${ }^{8-11}$, así como también los grupos de drogas más utilizados ${ }^{12,13}$.

Contrariamente a lo esperado, no se encontró relación significativa entre la utilización de fármacos del grupo A02 y los AINEs. Aunque fue elevada la proporción de pacientes que estando tratados con AINEs tenían indicación de A02, podría considerarse insuficiente si se tiene en cuenta que la población estudiada estaba expuesta a varios factores de riesgo para el desarrollo de enfermedades que cursan con hipersecreción ácida tales como edad, plurimedicación y medicación con AINEs, entre otros ${ }^{14,15}$. Aún desconociendo la existencia de otros factores de riesgo asociados a las patologías de base, puesto que el diseño del estudio no contemplaba la consulta de las historias clínicas, es llamativo que aproximadamente el $30 \%$ de los pacientes consumidores de AINEs no tuviesen prescripto ningún agente antiácido-antiulceroso. Se encuentran evidencias en la literatura científica de subutilización de protección gástrica en pacientes con factores de riesgo gastrointestinal ${ }^{16}$.

Por otra parte, atendiendo a que se encontró una fuerte asociación entre la utilización de analgésicos no AINEs y de fármacos del grupo A02 $\mathrm{y}$ a que fue significativamente mayor el número promedio de fármacos por pacientes en el grupo que utilizaba paracetamol y/o analgésicos opioides comparado con aquellos que utilizaron AINEs, es razonable pensar que en los consumidores de mayor número de fármacos se ha reemplazado la indicación de AINEs por analgésicos no antiinflamatorios. Si bien ante una mayor fragilidad orgánica y requerimiento de analgesia resultan convenientes los opioides, la prescripción de éstos podría estar condicionada por una mayor multiplicidad farmacológica o la mencionada posible subutilización de protección gástrica, factores conocidos como predisponentes de lesiones digestivas $^{14,15}$.

El AASp fue utilizado para la prevención primaria de eventos cardiacos, aunque se carece de evidencia riesgo-beneficio en pacientes de 80 años o más, se recomienda usarlo con precaución en este grupo etario ${ }^{6}$.

Es conocido que los ancianos institucionalizados suelen consumir más fármacos que los no institucionalizados ${ }^{12,17,18}$ y que presentan mayor prevalencia de patologías que comprometen al $\mathrm{SNC}^{19}$. La proporción de adultos mayores que utilizaron fármacos psicotrópicos se halla en el orden de la reportada ${ }^{20,21}$. El presente trabajo pone en evidencia una fuerte asociación entre polifarmacia y consumo tanto de benzodiazepinas como de psicofármacos en general ya que la prevalencia de utilización de estas drogas en el grupo con 6 o más medicamentos diarios duplicó a la del grupo con menos de seis.

A diferencia de otros trabajos, en este se encontró que los psicotrópicos prevalentes fueron las benzodiazepinas ${ }^{20,21} \mathrm{y}$ entre ellas el clonazepam. Ya se ha reportado en nuestro medio la gran extensión del uso de clonazepam como fármaco ansiolítico ${ }^{22}$. Deberían realizarse estudios más profundos para discernir entre lo que parece constituirse en una cultura prescriptiva y la existencia de factores que justifiquen su elevado consumo. Las benzodiazepinas, en especial las de vida media prolongada, pueden producir dependencia, daño cognitivo y aumento del riesgo de caídas en los adultos mayores, razón por la que forman parte del grupo de medicamentos cuyo uso debería evitarse en este sector de la población ${ }^{23-25}$.

En el grupo de pacientes con un número de fármacos inferior a 6 , los ancianos de menor edad, entre 60 y 79 años, utilizaban mayor cantidad de fármacos psicotrópicos y en particular de 
benzodiazepinas, que sus pares de 80 años o más. Puede interpretarse que los ancianos más jóvenes y aparentemente más autónomos fuesen más demandantes que sus pares mayores y estuviesen más expuestos a las presiones de medicalización que se evidencian en la sociedad en su conjunto ${ }^{26,27}$.

$\mathrm{Al}$ igual que en otros estudios, se encontró una fuerte asociación entre polimedicación y consumo de $\mathrm{MPI}^{8,28,29}$. En cambio, la prevalencia de MPI resultó más elevada que la reportada ${ }^{8,9,11,28,29}$. Puesto que se utilizaron los Criterios de Beers actualizados en 2012 y que al momento de la presentación de este trabajo no se publicaron estudios similares basados en ellos, las comparaciones no resultan adecuadas.

La no disponibilidad de las historias clínicas constituye una limitación importante para la interpretación apropiada del uso de algunos fármacos. Aún así, este estudio resulta valioso para reflexionar acerca de la conveniencia de utilizar protección gástrica en adultos mayores expuestos a factores de riesgo de lesiones gastrointestinales, así como también para reconsiderar la prescripción de ciertas drogas tales como las benzodiazepinas y los MPI.

El Hospital Geriátrico en el que se ha trabajado está sujeto a la utilización de un listado acotado de medicamentos que no está adaptado a las necesidades específicas de la tercera edad, lo cual resulta una restricción importante en la disponibilidad de fármacos con la que cuentan los profesionales prescriptores para la selección de una mejor opción terapéutica.

Puesto que el estudio se ha realizado en una Institución Geriátrica de referencia a nivel provincial, es posible extender los resultados de manera confiable a aquellos efectores que asisten a ancianos con características semejantes a las del grupo estudiado, como así también de sentar las bases para diseñar estrategias que permitan optimizar la calidad prescriptiva y consecuentemente la asistencial en la población de adultos mayores.

Agradecimientos: A la Dirección, al Servicio de Farmacia y a la Comunidad Asistencial del Hospital Geriátrico Provincial de Rosario por la colaboración prestada para la realización del presente trabajo. Al Gobierno de la Provincia de Santa Fe y a la Secretaría de Extensión de la Universidad Nacional de Rosario por el apoyo financiero brindado al proyecto.

\section{Referencias}

1. Montamat SC, Cusack B. Overcoming problems with polypharmacy and drug misuse in the elderly. Clin Geriatr Med 1992; 8: 143-58.

2. Gurwitz JH, Field TS, Harrold LR, Rothschild J, Debellis $\mathrm{K}$, Seger AC, et al. Incidence and preventability of adverse drug events among older persons in the ambulatory setting. JAMA 2003; 289: 1107-16.

3. Gray SL, Mahoney JE, Blough DK. Adverse drug events in elderly patients receiving home health services following hospital discharge. Ann Pharmacother 1999; 33: 1147-53.

4. Cannon KT, Choi MM, Zuniga MA. Potentially inappropriate medication use in elderly patients receiving home health care: A retrospective data analysis. Am J Geriatr Pharmacother 2006; 4: 134-43.

5. Laroche ML, Charmes JP, Merle L. Potentially inappropriate medications in the elderly: a French consensus panel list. Eur J Clin Pharmacol 2007; 63: 725-31.

6. The American Geriatrics Society 2012 Beers Criteria Update Expert Panel. AGS updated Beers Criteria for potentially inappropriate medication use in older adults. J Am Geriatr Soc 2012; 60: 616-31.

7. World Health Organization. ATC Index with DDDs and guidelines for ATC classification and DDD assignment, January 2004. Disponible en: http://www.who.org. [Consultado: 16 de agosto de 2012].

8. Fialova D, Topinkova E, Gambassi G, Finne-Soveri H, Jónsson PV, Carpenter I, et al, for the AdHOC Project Research Group. Potentially inappropriate medication use among elderly home care patients in Europe. JAMA 2005; 293: 1348-58.

9. Pattanaworasate W, Emmerton L, Pulver L, Winckel $\mathrm{K}$. Comparison of prescribing criteria in hospitalised Australian elderly. Pharmacy Practice 2010; 8: 13238.

10. Bergman A, Olsson J, Carlsten A, Waern M, Fastbom J. Evaluation of the quality of drug therapy among elderly patients in nursing homes. A computerized pharmacy register analysis. Scandinavian Journal of Primary Health Care 2007; 25: 9-14.

11. Roth M, Ivey J. Self-reported medication use in community-residing older adults: A pilot study. Am J Geriatric Pharmacother 2005; 3: 196-204.

12. Ramage-Morin PL. Medication use among senior Canadians. Statistics Canada, Catalogue no 82-003- XPE. Health Reports. Vol. 20, no 1, March 2009.

13. Valderrama Gama E, Rodríguez Artalejo F, Palacios Díaz A, Gabarre Orús P, Pérez del Molino Martín J. Consumo de medicamentos en los ancianos: resultados 
de un estudio poblacional. Rev Esp Salud Pública 1998; 72: 209-19.

14. Kaviani MJ, Pirastehfar M, Azari A, Saberifiroozi M. Etiology and outcome of patients with upper gastrointestinal bleeding: a study from South of Iran. Saudi J Gastroenterol 2010; 16 (4): 253-9.

15. Valenzuela López MI, Gastón Morat JL, Melguizo Jiménez M, Valenzuela López MM, Bueno Cavanillas A. Intervenciones sanitarias en atención primaria que disminuyen la hospitalización por Ambulatory Care Sensitive Conditions en mayores de 65 años. Aten Primaria 2007; 39 (10): 525-34.

16. Sturkenboom MC, Burke TA, Dieleman JP, Tangelder MJ, Lee F, Goldstein JL. Underutilization of preventive strategies in patients receiving NSAIDs. Rheumatology (Oxford) 2003; 42: 23-31.

17. Somers M, Rose E, Simmonds D, Whitelaw C, Calver J, Beer C. Quality use of medicines in residential aged care. Australian Family Physician 2010; 39: 413-17.

18. Kersten H, Ruths S, Wyller TB. Pharmacotherapy in nursing homes. Tidsskr Nor Laegeforen 2009; 129: 1732-5.

19. Valenzuela E, Martínez G. Uso de fármacos en las personas mayores. En: Marín PP, Gac H, Carrasco M, editores. Geriatría y Gerontología. 1a edición. Ediciones Universidad Católica de Chile-Grupo Guía Editores; 2008. p. 230-39.

20. Mann E, Köpke S, Haastert B, Pitkälä K, Meyer G. Psychotropic medication use among nursing home residents in Austria: a cross-sectional study. BMC Geriatr 2009; 9: 18.

21. Bhattacharjee S, Karkare SU, Kamble P. Aparasu RR.
Psychotropic drug utilization among elderly nursing home residents in the United States. Psychiatr Serv 2010; 61: 655 .

22. Quaglia Planas NB, Paciaroni J, Elías MM, Leiva M. Benzodiazepines use in a commune in the Rosario Metropolitan Area, Santa Fe Province, Argentina. Aten Primaria 2009; 41: 520-1.

23. Chutka DS, Takahashi PY, Hoel RW, Inappropriate medications for elderly patients. Mayo Clin Proc 2004; 79: 122-39.

24. Baranzini F, Diurni M, Ceccon F, Poloni N, Cazzamalli $S$, et al. Fall-related injuries in a nursing home setting: is polypharmacy a risk factor? BMC Health Serv Res 2009; 9: 228.

25. Gac H, Marín P, Castro S, Hoyl T, Valenzuela E. Caídas en adultos mayores institucionalizados: Descripción y evaluación geriátrica. Rev Med Chile 2003; 131: 887-94.

26. Orueta Sánchez R, Santos Rodríguez C, González Hidalgo E, Fagundo Becerra EM, Alejandre Lázaro G, Carmona de la Morena J, et al. Medicalización de la vida (I). Rev Clin Med Fam 2011; 4 (2): 150-61.

27. Barros JA. Nuevas tendencias de la medicalización. Ciênc. Saúde coletiva 2008; 13 (supl): 579-87.

28. Blasco Patino F, Pérez Maestu R, Martínez López de Letona J, Jiménez AI, García Navarro MJ. Estudio del consumo de fármacos inadecuados o no indicados en el anciano que ingresa en un Servicio de Medicina Interna. An Med Interna (Madrid) 2008; 25: 269-74.

29. Holguín-Hernández E, Orozco-Díaz J. Medicación potencialmente inapropiada en ancianos en un hospital de primer nivel, Bogotá 2007. Rev. Salud Pública 2010; 12 (2): 287-99. 